OPEN ACCESS

Edited by: Kenneth K.W. To,

The Chinese University of Hong Kong, China

Reviewed by:

Gopi Sundaram,

Central Food Technological Research

Institute (CSIR), India

Stefan Urbanski

University of Calgary, Canada

${ }^{*}$ Correspondence:

Jun Li

2307016@zju.edu.cn

tThese authors have contributed equally to this work

Specialty section:

This article was submitted to Gastrointestinal Cancers,

a section of the journal

Frontiers in Oncology

Received: 30 November 2020 Accepted: 17 February 2021

Published: 29 April 2021

Citation:

Kong X-X, Li X-L, Tian Y, Ye Q-C Xu X-M, Liu Y, Yang $Q$, Zhang L-N,

Mei Y-X, Wen J-H, Xiao Q, Li J-S,

Ding K-F and Li J (2021) The

Clinicopathological Characteristics of

Alpha-Fetoprotein-Producing

Adenocarcinoma of the

Gastrointestinal Tract-A

Single-Center Retrospective Study.

Front. Oncol. 11:635537.

doi: 10.3389/fonc.2021.635537

\section{The Clinicopathological} Characteristics of Alpha-Fetoprotein-Producing Adenocarcinoma of the Gastrointestinal Tract-A Single-Center Retrospective Study

\author{
Xiang-Xing Kong ${ }^{1,2+}$, Xin-Lin $\mathrm{Li}^{1,2,3+}$, Yu Tian ${ }^{4+}$, Qian-Cheng Ye ${ }^{4}$, Xiao-Ming Xu ${ }^{2,5}$, Yue Liu ${ }^{1,2}$, \\ Qi Yang ${ }^{2,5}$, Li-Na Zhang ${ }^{1,2}$, Yan-Xia Mei ${ }^{1,2}$, Ji-Hang Wen ${ }^{1,2}$, Qian Xiao ${ }^{1,2}$, Jing-Song Li ${ }^{4,6}$, \\ Ke-Feng Ding ${ }^{1,2}$ and Jun $L i^{1,2 *}$ \\ ${ }^{1}$ Department of Colorectal Surgery and Oncology, Key Laboratory of Cancer Prevention and Intervention, Ministry of \\ Education, The Second Affiliated Hospital, Zhejiang University School of Medicine, Hangzhou, China, ${ }^{2}$ Zhejiang University \\ Cancer Center, Hangzhou, China, ${ }^{3}$ Department of Breast Surgery, Hwa Mei Hospital, University of Chinese Academy of \\ Sciences, Ningbo, China, ${ }^{4}$ Engineering Research Center of EMR and Intelligent Expert System, Ministry of Education, College \\ of Biomedical Engineering and Instrument Science, Zhejiang University, Hangzhou, China, ${ }^{5}$ Department of Pathology, Key \\ Laboratory of Cancer Prevention and Intervention, Ministry of Education, The Second Affiliated Hospital, Zhejiang University \\ School of Medicine, Hangzhou, China, ${ }^{6}$ Research Center for Healthcare Data Science, Zhejiang Lab, Hangzhou, China
}

Alpha-fetoprotein (AFP)-producing adenocarcinoma from the gastrointestinal tract (APA-GI) is a rare type of highly malignant tumor with a poor prognosis. It may originate from any site along the Gl tract with similar clinicopathological characteristics. As limited research had ever described the characteristics of APA-GI, the present article intends to systemically investigate the clinicopathological characteristics of APA-Gl from a single center's retrospective study to deepen the understanding of the disease. A total of 177 patients pathologically diagnosed with APA-Gl between 2010 and 2017 at the Second Affiliated Hospital of Zhejiang University, School of Medicine, were included. Also, clinical data of 419 gastric cancers and 609 colorectal cancers from The Cancer Genome Atlas database were also extracted. Clinical information of patients from Second Affiliated Hospital of Zhejiang University, School of Medicine, was collected, and a median follow-up of 14.5 months was performed to investigate clinical characteristics of APA-GI. For the pathological characteristics of APA-GI, hematoxylin-eosin sections were reviewed, and immunohistochemistry of AFP was performed. The results showed that the primary tumor could develop through the whole Gl tract, including the esophagus (0.6\%), stomach (83.1\%), duodenum (1.1\%), ileum (0.6\%), appendix (0.6\%), colon (5.1\%), and rectum (7.9\%). Hepatoid adenocarcinoma is the main pathological feature of APA-Gl. AFP expression level in tumor tissue was not strictly associated with serum AFP or hepatoid differentiation. The prognosis of APA-Gl was worse than that of common adenocarcinoma of the Gl tract and liver metastasis, and high AFP levels suggest poor prognosis in patients with APA-GI. Therefore, the present study was the first 
research to systemically explore the clinicopathological characteristics of APA-GI. APA-GI occurs through the whole Gl tract with a significantly worse prognosis than common adenocarcinoma of GI. APA-Gl should be regarded as one kind of disease for its similar clinicopathological characteristics within patients.

Keywords: AFP-producing, gastrointestinal, adenocarcinoma, clinical characteristic, pathological characteristic

\section{INTRODUCTION}

Alpha-fetoprotein (AFP) is a glycoprotein produced by the fetal liver, yolk sac, and fetal gastrointestinal cells. Its content in adult serum is extremely low, and its abnormal increase is mainly found in hepatocellular carcinoma and yolk sac-derived tumors (1). However, abnormally elevated serum AFP is also reported in some kinds of adenocarcinoma, which are called AFP-producing adenocarcinoma (APA) (2). APA is a rare type of highly malignant tumor with a fairly poor prognosis $(3,4)$. It can originate from a variety of organs and mainly occurs in the digestive tract. The majority of APAs are developed in the stomach, accounting for $1.3-15 \%$ of all gastric cancers (GC) and $<1 \%$ of all colorectal cancers (CRCs) $(5,6)$. However, much less is known about the clinicopathological characteristics of APA.

The serum AFP in APA patients is increased $(>20 \mathrm{ng} / \mathrm{ml})$ and markers representing embryonic stem cells and hepatocellular carcinoma in tumor tissues, such as AFP protein, glypican3, Sal-like protein-4, and/or hepatocyte antigen-1, are positive $(7,8)$. APA originated from the gastrointestinal tract (APAGI) was reported to have some common clinical characteristics, such as early multi-organ metastasis, multidrug resistance, rapidly worsen after diagnosis, and much inferior prognosis than common adenocarcinoma of the gastrointestinal tract (CA-GI) (5, 6, 9-11). Although the previous studies of APA-GI usually only included APA-GC or APA-CRC $(3,12)$, we found APAGI could also originate from the whole GI tract, including the esophagus, small intestinal, and appendix.

Therefore, we believe that APA-GI is a separate class of disease that showed similar clinicopathological characteristics within different origins. As the research on APA-GI is limited, how to accurately differentiate it from CA-GI and get accurate treatment to improve the prognosis is still in challenge $(2,13)$. The present article intends to systemically investigate the clinical and pathological characteristics of APA-GI from a single center's retrospective study to deepen the understanding of the disease.

\section{PATIENTS AND METHODS}

\section{Patients From the Second Affiliated Hospital of Zhejiang University, School of Medicine}

Patients pathologically diagnosed with APA-GI between 2010 and 2017 at the Second Affiliated Hospital of Zhejiang University, School of Medicine (SAHZU) were included in the study. Also, the exclusion criteria were the following: (1) pregnancy status; (2) synchronously diagnosed with acute or chronic hepatitis, liver cirrhosis, hepatocellular carcinoma, teratoma, and germinoma, or (3) cancer history other than GI adenocarcinoma. APA-GI was defined as follows: (1) pathologically confirmed primary gastrointestinal adenocarcinoma and (2) serum AFP $>20 \mathrm{ng} / \mathrm{ml}$ during the whole course. Two pathologists reviewed all hematoxylin-eosin (HE) sections to exclude the yolk sac tumorlike area or neuroendocrine component.

\section{Patients From the Cancer Genome Atlas}

A total of 419 GC patients and 609 CRC patients from The Cancer Genome Atlas (TCGA) database (URL: http://cancergenome. nih.gov) were included. All these patients have pathologically confirmed adenocarcinoma.

\section{Data Collection and Follow-Up}

For patients from SAHZU, clinical characteristics were all collected from the hospital information system, which included age, sex, primary tumor location, tumor size, pathological type, differentiated level, tumor-node-metastasis (TNM) staging, metastasis status, operation method, operation time, post operation pathology, serum AFP level, and treatment strategy. The last follow-up date was January 1, 2019. The major approach for follow-up was telephone calls or outpatient visits.

For patients from TCGA, we extracted patients' sex, pathology, TNM staging, survival time, survival status, and via R.

\section{Immunohistochemistry}

Immunohistochemistry (IHC) staining was performed according to the standard protocol. Freshly cut $4-\mu \mathrm{m}$ paraffin-embedded sections were incubated overnight at $62^{\circ} \mathrm{C}$ and then deparaffinized by xylene and dehydrated with ethanol. Antigen retrieval was performed using a pressure cooker in citrate antigen retrieval solution $(\mathrm{pH}$ 8.0) for $15 \mathrm{~min}$, then incubated with $3 \%$ peroxide for $10 \mathrm{~min}$ and blocked by nonspecific staining blocking reagent (Dako, Glostrup, Denmark). Staining was performed with AFP polyclonal antibody (ab169552, Abcam). Briefly, $150 \mu \mathrm{l}$ of rabbit polyclonal anti-AFP antibody at $100 \times$ dilution was incubated for $120 \mathrm{~min}$, washed, subsequently incubated with horseradish peroxidase and anti-rabbit antibodyconjugated polymer for $30 \mathrm{~min}$, washed, and finally incubated with 3,3'diaminobenzidine substrate for signal development. Sections were at last counterstained with hematoxylin.

Two pathologists independently reviewed the IHC sections. The staining intensity was scored as 0 (negative), 1 (weak), 2 (moderate), or 3 (strong), whereas the staining extent was scored as $0(<5 \%), 1(5-25 \%), 2(26-50 \%), 3(51-75 \%)$, and $4(>75$ $\%)$ according to the positive staining area proportion. Scores for staining intensity and extent were then multiplied to generate the immunoreactivity score (IRS) for each case. Therefore, IRSs $0-$ $1,2-3,4-8$, and $9-12$ were referred to negative, week positive, 
moderately positive, and strongly positive, respectively. IRS $<4$ was termed as AFP low expression, whereas IRS $\geq 4$ was termed as AFP high expression.

\section{Statistical Analysis}

The chi-squared test and Fisher's exact analysis were performed to compare the clinical and pathological characteristics between groups. Non-Gaussian-distributed data were presented as medians and interquartile ranges and evaluated by the Kruskal-Wallis test. Survival data were presented by KaplanMeier survival curves and compared using the log-rank test. With the Cox proportional hazards model, univariate and multivariate survival analyses were conducted to identify independently significant variables. Multivariate analyses were all based on factors $<0.5$, which were tested by univariate analyses. A $P$-value of 0.05 or less indicated statistical significance. Statistical and graphical analyses were performed with SPSS 23.0 and GraphPad Prism 7 software.

\section{RESULTS}

\section{Clinical Characteristics of Alpha-Fetoprotein-Producing Adenocarcinoma From the Gastrointestinal Tract}

A total of 177 APA-GI patients were finally included. The median age was 63 years old (interquartile range, 53.5-71.0 years old), and males accounted for $69.5 \%$. The primary tumor could develop through the whole gastrointestinal tract, including the esophagus $(0.6 \%)$, stomach $(83.1 \%)$, duodenum (1.1\%), ileum $(0.6 \%)$, appendix $(0.6 \%)$, colon $(5.1 \%)$, and rectum (7.9\%). Meanwhile, it could also synchronously develop in two organs, such as the stomach and rectum (0.6\%) and colon and rectum $(0.6 \%)$. The pathological type of APA-GI included adenocarcinoma with high-, media-, and low-differentiated, signet-ring cell carcinoma and mucinous adenocarcinoma. Among 71 non-metastasis patients, $18.3 \%$ received operation, whereas $77.5 \%$ received a combination of treatments based on surgery. Within all APA-GI patients, $58.2 \%$ of patients present metastasis at the initial diagnosis, whereas 145 (81.9\%) of patients finally developed metastasis. The liver was the most common site of APA-GI metastasis with a rate of $53.1 \%$. Among gastric and colorectal APAs, $71(40.1 \%)$ and $11(47.8 \%)$ of patients were initially diagnosed with liver metastasis, respectively (Table 1).

Increased serum AFP characterizes APA-GI. We found the AFP-high group (AFP-H group, AFP $\geq 200 \mathrm{ng} / \mathrm{ml}$ ) had more stage IV $(P=0.009)$ and liver metastases $(P<0.001)$ when compared with the AFP-low group (AFP-L group, AFP < $200 \mathrm{ng} / \mathrm{ml}$ ). Additionally, the AFP-H group was characterized by larger tumor size $(P=0.047)$, a higher proportion of adenocarcinoma $(P=0.022)$, and hepatoid adenocarcinoma (HA) $(P=0.006)$ (Table 2).
TABLE 1 | Baseline Characteristics of APA-GI.

\begin{tabular}{|c|c|}
\hline Characteristic & Total \\
\hline Age(y), median(IQR) & $63.0(53.5-71.0)$ \\
\hline Sex, Male/Female (Male\%) & 123/54 (69.5) \\
\hline \multicolumn{2}{|l|}{ Primary site, $n$ (\%) } \\
\hline Stomach & $147(83.1)$ \\
\hline Colon & $9(5.1)$ \\
\hline Rectum & $14(7.9)$ \\
\hline Appendix & $1(0.6)$ \\
\hline Duodenum & $2(1.1)$ \\
\hline Ileum & $1(0.6)$ \\
\hline Esophagus & $1(0.6)$ \\
\hline Stomach and rectum & $1(0.6)$ \\
\hline Colon and rectum & $1(0.6)$ \\
\hline Tumor size(cm), median(IQR) & $4.5(3.1-5.5)$ \\
\hline $\begin{array}{l}\text { Pathological type, } n(\%) \\
\text { Adenocarcinoma }\end{array}$ & $147(83.1)$ \\
\hline Signet ring cell carcinoma & $20(11.3)$ \\
\hline Mucinous adenocarcinoma & $10(5.6)$ \\
\hline \multicolumn{2}{|l|}{ Differentiation, $\boldsymbol{n}(\%)$} \\
\hline Highly differentiated & $3(1.7)$ \\
\hline Moderately differentiated & $43(24.3)$ \\
\hline Poorly differentiated & $109(61.6)$ \\
\hline Default & $22(12.4)$ \\
\hline \multicolumn{2}{|l|}{ pT, $n(\%)$} \\
\hline 1 & $8(8.2)$ \\
\hline 2 & $12(12.4)$ \\
\hline 3 & $12(12.4)$ \\
\hline 4 & $58(59.8)$ \\
\hline Default & $7(7.2)$ \\
\hline \multicolumn{2}{|l|}{$\mathrm{pN}, \boldsymbol{n}(\%)$} \\
\hline 0 & $20(20.6)$ \\
\hline 1 & $17(17.5)$ \\
\hline $2-3$ & $50(51.5)$ \\
\hline Default & $10(10.3)$ \\
\hline \multicolumn{2}{|l|}{ TNM staging, $\boldsymbol{n}(\%)$} \\
\hline 1 & $8(4.5)$ \\
\hline ॥ & $17(9.6)$ \\
\hline III & $46(26.0)$ \\
\hline IV & $103(58.2)$ \\
\hline Default & $3(1.7)$ \\
\hline \multicolumn{2}{|l|}{ Metastatic site ${ }^{\#}$ n (\%) } \\
\hline Liver & $94(53.1)$ \\
\hline Lymph node & $71(40.1)$ \\
\hline Lung & $19(10.7)$ \\
\hline Peritoneal cavity & $16(9.0)$ \\
\hline Bone & $15(8.5)$ \\
\hline Adrenal gland & $7(4.0)$ \\
\hline Pelvic cavity & $4(2.3)$ \\
\hline Spleen & $4(2.3)$ \\
\hline Pancreas & $4(2.3)$ \\
\hline Ovary and fallopian tube & $4(2.3)$ \\
\hline Brain & $3(1.7)$ \\
\hline
\end{tabular}


TABLE 1 | Continued

\begin{tabular}{lc}
\hline Characteristic & Total \\
\hline Lymphatic or/and blood vessel invasion & $39(40.2)$ \\
Yes & $38(39.2)$ \\
No & $20(20.6)$ \\
Default & \\
Nerve invasion & $26(26.8)$ \\
Yes & $54(55.7)$ \\
No & $17(17.5)$ \\
Default & \\
Hepatoid Adenocarcinoma* & $34(34 \%)$ \\
Yes & $66(66 \%)$ \\
No & \\
Treatments & $46(26.0)$ \\
Chemotherapy & $17(9.6)$ \\
Surgery & $90(50.8)$ \\
Comprehensive treatment & $24(13.5)$ \\
Other(Symptomatic treatment & \\
?Chinese herbology) & \\
\hline
\end{tabular}

\#, For patients with multiple metastases, all the metastatic foci of different parts were included separately; *, Only 100 patients receiving second pathological diagnosis were included.

\section{Hepatoid Adenocarcinoma Is the Main \\ Pathological Feature of \\ Alpha-Fetoprotein-Producing Adenocarcinoma From the Gastrointestinal Tract}

Two pathologists reviewed the $100 \mathrm{HE}$ sections available. Thirty-four sections were reported (32 surgical samples and 2 endoscopic samples) containing HA regions. However, only three of these were first diagnosed as HA. Similar to primary hepatocellular carcinoma, HA consists of hepatoid cells with abundant blood sinuses. Cancer cells from HA often present with round nuclei, hyper-chromatin, prominent nucleoli, and clear cytoplasm. HA is also usually nested or banded and can be easily confused with normal adenocarcinoma (Figure 1).

We further compared the clinical characteristics between HA and non-HA within APA-GI patients. Seventy-three patients who received the operation were included. Compared with the non-HA group (41 cases), the HA group (32 cases) had more male patients $(P=0.002)$ and a higher proportion of low-differentiation $(P=0.007)$ (Table 3). However, the 5-year survival was insignificant between these two groups (non-HA and HA groups: 46.9 vs. $18.5 \%$, respectively, $P=0.0953$, Supplementary Figure 1).

\section{Alpha-Fetoprotein-Producing Was Not Strictly Expressed in Alpha-Fetoprotein-Producing Adenocarcinoma From the Gastrointestinal Tract Patients' Tumor Tissue}

IHC was performed in 99 tumor specimens to explore the AFP expression in APA-GI patients. IRS was then evaluated by two
TABLE 2 | Comparison of clinical baseline characteristics of APA-GI patients with different serum AFP levels.

\begin{tabular}{|c|c|c|c|}
\hline Characteristic & AFP-L & AFP-H & $P$ value \\
\hline Age(y), median(IQR) & $63.0(52.0-72.0)$ & $63.0(57.0-69.0)$ & 0.814 \\
\hline Sex, Male/Female (Male\%) & 73/35 (67.6) & $50 / 19(72.5)$ & 0.492 \\
\hline Primary site, $n(\%)$ & & & 0.596 \\
\hline Stomach & $88(81.5)$ & $59(85.5)$ & \\
\hline Large intestine & $17(15.7)$ & $7(10.1)$ & \\
\hline Small intestine & $1(0.9)$ & $2(2.9)$ & \\
\hline Esophagus & $1(0.9)$ & $0(0)$ & \\
\hline Multiple primary sites & $1(0.9)$ & $1(1.4)$ & \\
\hline Tumor size(cm), median(IQR) & $4.0(3.0-5.0)$ & $5.0(4.0-6.0)$ & 0.047 \\
\hline Pathological type, $n$ (\%) & & & 0.022 \\
\hline Adenocarcinoma & $83(76.9)$ & $64(92.8)$ & \\
\hline Signet ring cell carcinoma & $17(15.7)$ & $3(4.3)$ & \\
\hline Mucinous adenocarcinoma & $8(7.4)$ & $2(2.9)$ & \\
\hline Differentiation, $n$ (\%) & & & 0.473 \\
\hline Highly differentiated & $1(0.9)$ & $2(2.9)$ & \\
\hline Moderately differentiated & $27(25.0)$ & $16(23.2)$ & \\
\hline Poorly differentiated & $64(59.3)$ & $45(65.2)$ & \\
\hline Default & $16(14.8)$ & $6(8.7)$ & \\
\hline pT, $n(\%)$ & & & 0.524 \\
\hline 1 & 7 (10.6) & $1(3.2)$ & \\
\hline 2 & $10(15.2)$ & $2(6.5)$ & \\
\hline 3 & $8(12.1)$ & $4(12.9)$ & \\
\hline 4 & $37(56.1)$ & $22(71.0)$ & \\
\hline Default & $4(6.1)$ & $2(6.5)$ & \\
\hline $\mathrm{pN}, n(\%)$ & & & 0.643 \\
\hline 0 & 12 (18.2) & $8(25.8)$ & \\
\hline 1 & $13(19.7)$ & $4(12.9)$ & \\
\hline $2-3$ & $33(50.0)$ & $17(54.8)$ & \\
\hline Default & $8(12.1)$ & $2(6.5)$ & \\
\hline TNM staging, $n(\%)$ & & & 0.009 \\
\hline$|-|||$ & $51(47.2)$ & $20(29.0)$ & \\
\hline IV & $54(50.0)$ & 49 (71.0) & \\
\hline Default & $3(2.8)$ & $0(0.0)$ & \\
\hline Liver metastasis & & & $<0.001$ \\
\hline Yes & $41(38.0)$ & $53(76.8)$ & \\
\hline No & $67(62.0)$ & $16(23.2)$ & \\
\hline Extrahepatic metastasis & & & 0.962 \\
\hline Yes & $63(58.3)$ & $40(58.0)$ & \\
\hline No & 45 (41.7) & $29(42.1)$ & \\
\hline Lymphatic or/and blood vessel invasion & & & 0.509 \\
\hline Yes & $24(36.4)$ & $15(48.4)$ & \\
\hline No & $28(42.4)$ & $10(32.3)$ & \\
\hline Default & $14(21.2)$ & $6(19.4)$ & \\
\hline Nerve invasion & & & 0.126 \\
\hline Yes & $14(21.2)$ & $12(38.7)$ & \\
\hline No & $38(57.6)$ & $16(51.6)$ & \\
\hline Default & $14(21.2)$ & $3(9.7)$ & \\
\hline Hepatoid Adenocarcinoma\# & & & 0.006 \\
\hline Yes & $16(32.7)$ & $16(66.7)$ & \\
\hline No & $33(67.3)$ & 8 (33.3) & \\
\hline
\end{tabular}

\#, Considering the lack of pathological sample obtained from endoscopy, 73 surgical patients out of 100 patients receiving second pathological diagnosis were included. 


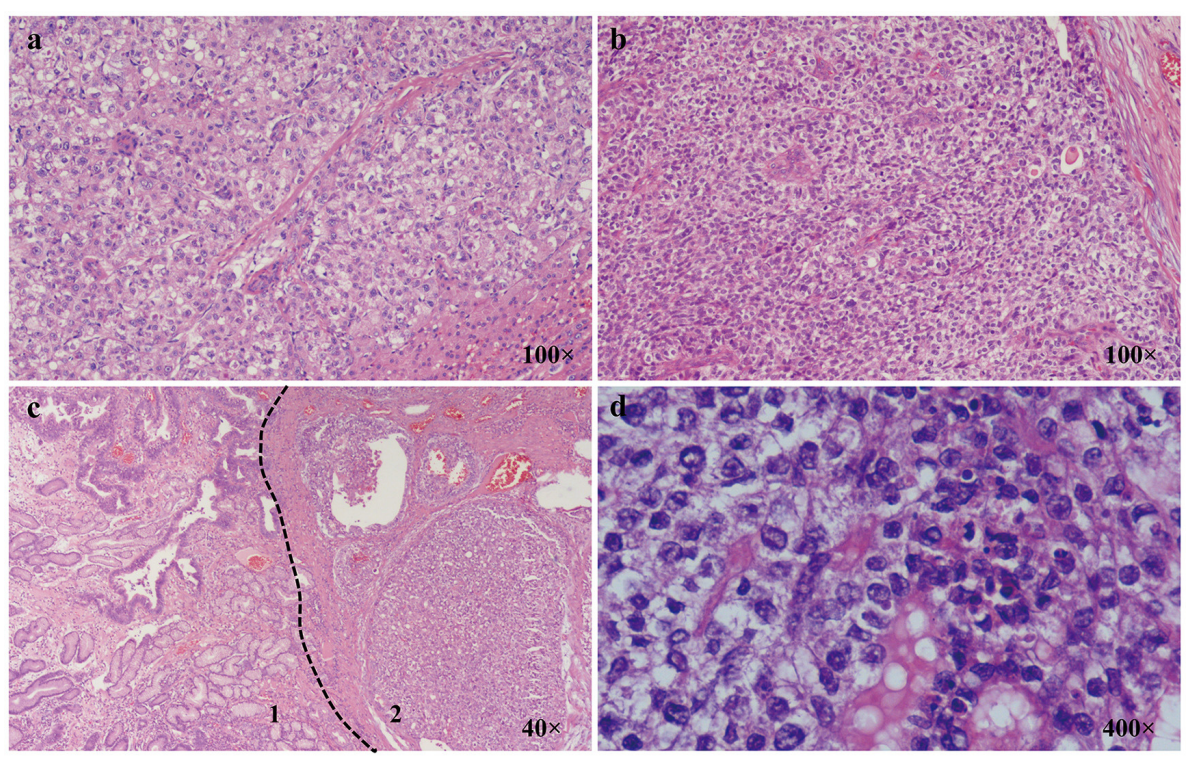

FIGURE 1 | Characteristics of AFP-GI under a light microscope. (a) Classic primary hepatic carcinoma (100x); (b) Classic hepatoid adenocarcinoma (100x); (c) 1. Zone of adenocarcinoma with moderately differentiation; 2 . Zone of adenocarcinoma with hepatoid differentiation (40x); (d) Zone of adenocarcinoma with hepatoid differentiation is composed of cells with round nuclei, coarse chromatin, obvious nucleoli, and clear cytoplasm (400x).

pathologists independently (Supplementary Figure 2). There were 15 AFP high expression patients, and the other 84 patients were classified as AFP low expression. AFP was positive in $36.4 \%$ of APA-GI patients, and it was also positive in $43.3 \%$ of patients with elevated serum AFP before surgery. Interestingly, AFP was only partly expressed in HA with a positive rate of $73.5 \%$. Among the 36 patients with positive IHC AFP, 5.6\% had normal levels of AFP before surgery. Therefore, the AFP expression level in tumor tissue was not strictly associated with serum AFP or hepatoid differentiation. Additionally, for 73 patients who received the operation, 35 patients had neither positive AFP IHC nor HA (AFP-/HA-), whereas the others performed at least one characteristic. However, the 5-year survival between these two groups was similar ( 44.54 vs. $24.26 \%, P=0.342$, Supplementary Figure 3).

\section{Prognosis of Alpha-Fetoprotein-Producing Adenocarcinoma From the Gastrointestinal Tract Was Worse Than That of Common Adenocarcinoma of the Gastrointestinal Tract}

To investigate the prognosis of APA-GI, a median follow-up of 14.5 months was performed. There were only 31 (17.5\%) who survived, whereas 129 (72.9\%) died and 17 (9.6\%) lost followup among the patients included. The 1-, 3-, and 5-year overall survival rates were $50.6,28.5$, and $17.7 \%$, respectively.

To further compare the prognosis between APA-GI and CA, we extracted the follow-up information of CA-GC and CA-CRC from TCGA. For this reason, only 154 APA-GC and APA-CRC patients of 177 APA-GI patients from our center were analyzed. There was no significant difference in the 5-year survival between stages I-III APA-GC and CA-GC (28.8 vs. 41.9\%, respectively, $P=0.357)$. However, the prognosis of APA-GC in stage IV was significantly worse than that of CA-GC (4.4 vs. $32.1 \%$, $P<0.001)$. The 5-year survival of patients with APA-CRC was similar to CA-CRC (5-year survival: stages I-III APA-CRC and CA-CRC, 71.32 vs. $70 \%$, respectively, $P=0.132$; stage IV APA-CRC and CA-CRC, 13.89 vs. $26.73 \%$, respectively, $P=$ 0.091). After combining CRC and GC, survival analysis of APAGI from our center showed significantly worse 5-year survival than that of CA-GI from TCGA (stages I-III, 32.8 vs. $59.4 \%$, respectively, $P<0.001$; stage $\mathrm{IV}, 6.3$ vs. $30.1 \%$, respectively, $P$ $<0.001$; stages I-IV, 17.7 vs. $55.9 \%$, respectively, $P<0.001$ ) (Figure 2).

\section{Liver Metastasis and High Alpha-Fetoprotein-Producing Levels Suggest Poor Prognosis in Patients With Alpha-Fetoprotein-Producing Adenocarcinoma From the Gastrointestinal Tract}

Liver metastasis was found to be an independent risk factor for total survival in APA-GI patients after multivariate Cox regression analysis (hazard ratio 3.59, 95\% confidence interval 1.28-10.02, $P=0.015$ ) (Table 4). To investigate the impact of liver metastasis-free survival, we further conducted Cox multivariate regression analysis on the data, including the level of AFP. We found that AFP $\geq 200 \mathrm{ng} / \mathrm{ml}$ was an independent risk factor for the liver metastasis of APA-GI (hazard ratio 4.55, 95\% confidence interval 1.39-14.87, $P=0.012$ ) (Table 5).

Kaplan-Meier curve that showed the prognosis of the AFP$\mathrm{H}$ group was significantly worse than that of the AFP-L group. 
TABLE 3 | Comparison of clinical baseline characteristics between APA-GI patients with $\mathrm{HA}$ and non-HA.

\begin{tabular}{|c|c|c|c|}
\hline Characteristic & non-HA & HA & $P$-value \\
\hline Age(y), median(IQR) & $61.0(50.0-70.5)$ & $53.0(58.0-71.8)$ & 0.247 \\
\hline Sex, Male/Female (Male\%) & $22 / 19(53.7)$ & 28/4 (87.5) & 0.002 \\
\hline Primary site, $n(\%)$ & & & 0.321 \\
\hline Stomach & $33(80.5)$ & $27(84.4)$ & \\
\hline Large intestine & $7(17.1)$ & $3(9.4)$ & \\
\hline Small intestine & $1(2.4)$ & $0(0.0)$ & \\
\hline Esophagus & $0(0.0)$ & $1(3.1)$ & \\
\hline Multiple primary sites & $0(0.0)$ & $1(3.1)$ & \\
\hline Tumor size(cm), median(IQR) & $4.5(3.5-5.3)$ & $5.0(3.0-6.0)$ & 0.668 \\
\hline Differentiation, $n$ (\%) & & & 0.007 \\
\hline Highly differentiated & $0(0.0)$ & $0(0.0)$ & \\
\hline Moderately differentiated & $17(41.5)$ & $5(15.6)$ & \\
\hline Poorly differentiated & $21(51.2)$ & $27(84.4)$ & \\
\hline Default & $3(7.3)$ & $0(0.0)$ & \\
\hline $\mathrm{pT}, n(\%)$ & & & 0.913 \\
\hline 1 & $3(7.3)$ & $2(6.3)$ & \\
\hline 2 & $6(14.6)$ & $4(12.5)$ & \\
\hline 3 & $5(12.2)$ & $6(18.8)$ & \\
\hline 4 & 27 (65.9) & $20(62.5)$ & \\
\hline $\mathrm{pN}, n(\%)$ & & & 0.825 \\
\hline 0 & 8 (19.5) & 7 (21.9) & \\
\hline 1 & $6(14.6)$ & $7(21.9)$ & \\
\hline $2-3$ & $25(61.0)$ & $17(53.1)$ & \\
\hline Default & $2(4.9)$ & $1(3.1)$ & \\
\hline TNM staging, $n(\%)$ & & & 0.514 \\
\hline$|-|||$ & $31(75.6)$ & $22(68.8)$ & \\
\hline IV & $10(24.4)$ & $10(31.3)$ & \\
\hline Liver metastasis & & & 0.084 \\
\hline Yes & $9(22.0)$ & $13(40.6)$ & \\
\hline No & $32(78.0)$ & $19(59.4)$ & \\
\hline Extrahepatic metastasis & & & 0.756 \\
\hline Yes & $22(53.7)$ & $16(50.0)$ & \\
\hline No & $19(46.3)$ & $16(50.0)$ & \\
\hline Lymphatic or/and blood vessel invasion & & & 0.269 \\
\hline Yes & $19(46.3)$ & $19(59.4)$ & \\
\hline No & $22(53.7)$ & $13(40.6)$ & \\
\hline Nerve invasion & & & 0.486 \\
\hline Yes & $11(26.8)$ & $11(34.4)$ & \\
\hline No & $30(73.2)$ & $21(65.6)$ & \\
\hline
\end{tabular}

HA, hepatic adenocarcinoma; non-HA, non-hepatic adenocarcinoma.

The 5-year survival of AFP $\geq 200 \mathrm{ng} / \mathrm{ml}$ was $7.5 \%$, whereas it increased to $26.0 \%$ in the APA-GI patients with AFP < $200 \mathrm{ng} / \mathrm{ml}(P=0.002)$. The proportion of stage IV patients in the AFP-H and AFP-L groups were 71 and 50\%, respectively. The prognosis of stages I-III patients with an AFP $\geq 200 \mathrm{ng} / \mathrm{ml}$ was worse than that of AFP $<200 \mathrm{ng} / \mathrm{ml}$ (5-year survival: 11.3 vs. $44.4 \%$, respectively, $P=0.030$ ), whereas there was no statistical difference in the prognosis of stage IV patients (5-year survival: 3.5 vs. $5.6 \%$, respectively, $P=0.421$ ) (Figure 3 ).

\section{DISCUSSION}

APA is a rare kind of malignant tumor, which could originate from various organs, such as the lung, ovary, kidney, esophagus, stomach, small intestine, colorectal, and appendix (3, 10, 14-18). The present study focused on the clinicopathological features of APA-GI via a retrospective analysis. Data from SAHZU showed APA-GI was more likely to occur in middle-aged and older adults, and it could originate from the whole GI tract, especially in the stomach. The rate of tumor metastasis is high, and most patients were initially diagnosed with liver metastasis. The positive rate of AFP in APA-GI tumor tissue was 36.4\%, and it was partly expressed in hepatoid differentiated tissue. The prognosis of APA-GI was significantly worse than CA-GI, and patients with AFP $\geq 200 \mathrm{ng} / \mathrm{ml}$ were even worse in APA-GI. Liver metastasis was an independent risk factor for the overall survival of APA-GI, and patients with AFP $\geq 200 \mathrm{ng} / \mathrm{ml}$ were more like to develop liver metastasis. Ren et al. had also previously investigated the clinicopathological features and prognosis of APA-CRC, especially the tumor location and survival, which was with our research. Additionally, they divided APA-CRC patients into three histologic types: the common adenocarcinoma type, mucinous adenocarcinoma type, and hepatoid type (3). However, the present research still had some progress. We provided a sufficient sample size with 177 APA-GIs to reach statistically significant conclusions. We included not only APA-CRC but also other GI sites' APA to obtain a full landscape of APA-GI. In addition, we also described several risk factors of liver metastasis and prognosis.

Most APA-GIs were low differentiation. The tumor cells were distributed in clusters or bands, and the stroma was rich in blood sinuses. HA was the main pathological feature of APAGI. However, among 100 patients included, only three were reported HA in the original pathological diagnosis, whereas there were actually $34 \mathrm{HA}$ patients after reviewing all $\mathrm{HE}$ sections. The main reasons for missed diagnosis of APA-GI may be as follows. First, $\mathrm{HA}$ is often intermixed within CA components, making the former difficult to recognize, and it leads to the missed diagnosis of APA-GI. Secondly, HA is not necessary to report in routine pathological diagnosis, making pathologists pay insufficient attention. Although HA is the major pathologic type of APA-GI, only $30 \%$ of HA cases have been reported within APA-GI in our center. Therefore, the serum AFP index is still essential for the diagnosis of APA-GI. The current diagnostic criteria for HA proposed by the World Health Organization relies on pathological morphology without the need for IHC evidence (World Health Organization Classification of Tumours, 5th Edition, Volume 1. Digestive System Tumours. Lyon: IARC Press, 2019) (19). Therefore, the present study asked two pathologists to independently review HE sections to confirm hepatoid differentiation without additional Hepa1 and glypican-3 IHC. Additionally, the survival was similar between APA-GI with and without hepatoid differentiation, but both were worse than that of CA-GI. Therefore, using only hepatoid differentiation in the diagnosis of APA-GI will lead to missed diagnosis and inadequate understanding of prognosis. In previous studies, elevated serum AFP levels, positive AFP IHC, 


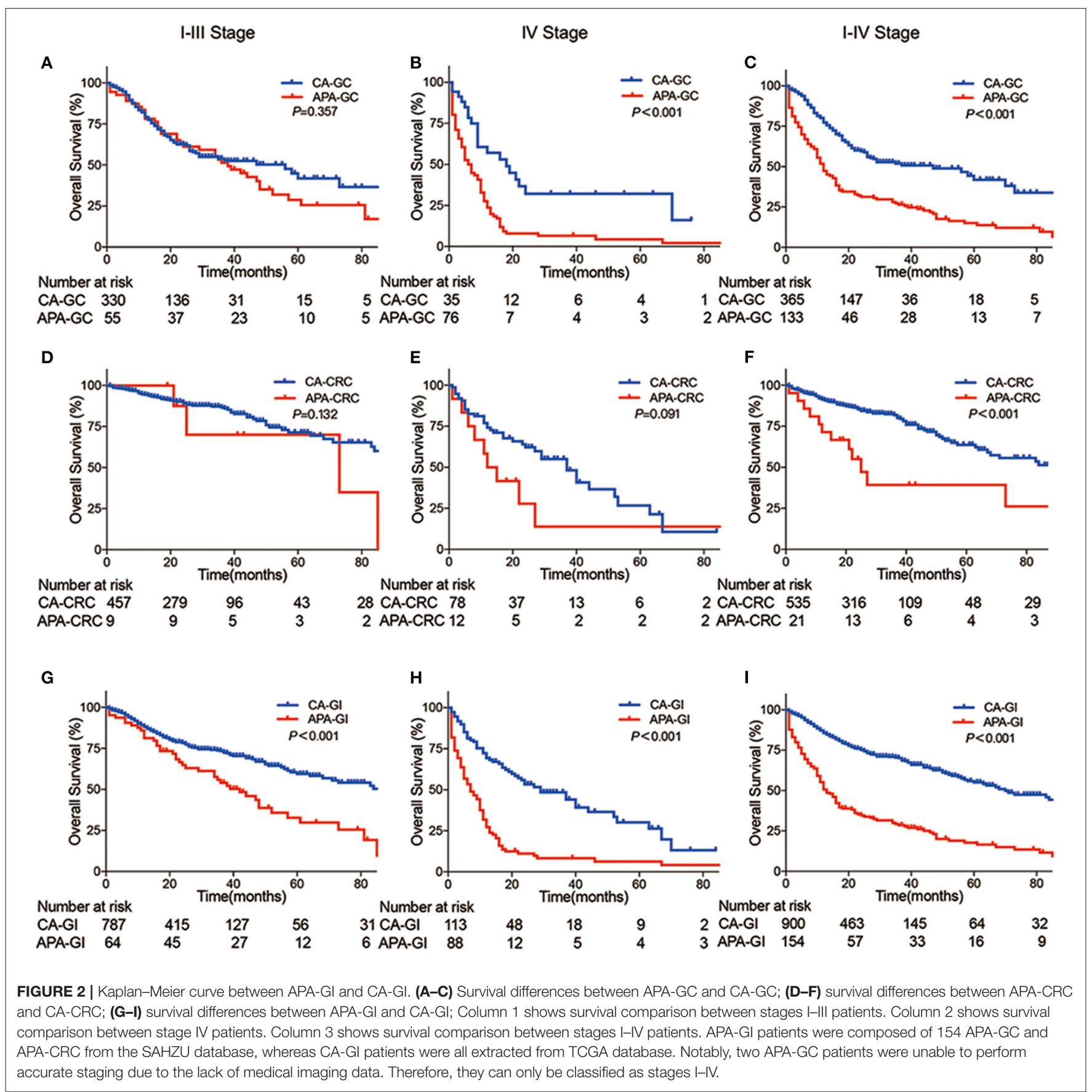

or HA detection was used as diagnostic criteria for APA-GI, but there is no standard criteria or consensus until now $(6,20-$ 22). In our study, neither HA nor positive AFP IHC was the sufficient condition of APA-GI diagnosis, as only $52 \%$ of patients existed either HA or positive AFP IHC. For these patients, their prognoses were similar to that of APA-GI patients with only elevated serum AFP. Therefore, we suggested APA-GI should be regarded as one kind of disease, and its diagnosis depends on both serum AFP level and pathology. Also, pathologists should pay more attention to hepatoid differentiation, and there should be more molecular pathology research to construct the diagnostic criteria of APA-GI.

High metastasis rate and liver metastasis are the main characteristics of APA-GI at initial diagnosis. Many studies have found that $12-25 \%$ of patients with digestive tract adenocarcinoma have metastases after initial diagnosis $(23,24)$. Nearly $40-50 \%$ of patients have metastases during the whole course of the disease $(24,25)$. Among them, $4-14 \%$ gastric 
TABLE 4 | Regression analysis for prognostic risk factors of APA-Gl.

\begin{tabular}{|c|c|c|c|c|}
\hline \multirow[t]{2}{*}{ Variable } & \multicolumn{2}{|c|}{ Single factor } & \multicolumn{2}{|c|}{ Multiple factors } \\
\hline & $\mathrm{HR}(95 \% \mathrm{Cl})$ & $P$-value & $\mathrm{HR}(95 \% \mathrm{Cl})$ & $P$-value \\
\hline Age, y & & 0.078 & & 0.913 \\
\hline$<60$ & 1 & & 1 & \\
\hline$\geq 60$ & $1.40(0.96-2.05)$ & & $1.05(0.42-2.62)$ & \\
\hline Sex & & 0.075 & & 0.551 \\
\hline Male & 1 & & 1 & \\
\hline Female & $0.70(0.47-1.04)$ & & $1.38(0.48-3.94)$ & \\
\hline AFP, ng/ml & & 0.005 & & 0.877 \\
\hline$<200$ & 1 & & 1 & \\
\hline$\geq 200$ & $1.67(1.17-2.38)$ & & $0.93(0.38-2.27)$ & \\
\hline Primary site & & 0.241 & & \\
\hline Stomach & 1 & & & \\
\hline Large intestine & $0.57(0.32-1.02)$ & & & \\
\hline Small intestine & $0.66(0.16-2.66)$ & & & \\
\hline Esophagus & $1.04(0.14-7.45)$ & & & \\
\hline Multiple primary sites & $0.26(0.04-1.86)$ & & & \\
\hline Tumor size, cm & & 0.028 & & 0.59 \\
\hline$<5$ & 1 & & 1 & \\
\hline$\geq 5$ & $1.99(1.08-3.65)$ & & $0.79(0.34-1.83)$ & \\
\hline Pathological type & & 0.660 & & \\
\hline Adenocarcinoma & 1 & & & \\
\hline Signet ring cell carcinoma & $0.78(0.44-1.37)$ & & & \\
\hline Mucinous adenocarcinoma & $0.88(0.45-1.75)$ & & & \\
\hline Differentiation & & 0.926 & & \\
\hline Highly differentiated & 1 & & & \\
\hline Moderately differentiated & $0.90(0.21-3.79)$ & & & \\
\hline Poorly differentiated & $0.84(0.20-3.42)$ & & & \\
\hline pT & & 0.056 & & 0.96 \\
\hline 1 & 1 & & 1 & \\
\hline 2 & $1.60(0.32-7.98)$ & & $1.52(0.15-15.72)$ & \\
\hline 3 & $2.68(0.49-14.73)$ & & $1.95(0.18-21.38)$ & \\
\hline 4 & $3.98(0.96-16.50)$ & & $1.60(0.18-14.01)$ & \\
\hline $\mathrm{pN}$ & & 0.028 & & 0.071 \\
\hline 0 & 1 & & 1 & \\
\hline 1 & $2.54(0.99-6.57)$ & & $2.80(0.79-9.90)$ & \\
\hline $2+3$ & $2.68(1.29-5.56)$ & & $3.05(1.16-8.06)$ & \\
\hline TNM staging & & $<0.001$ & & 0.486 \\
\hline$|-|||$ & 1 & & 1 & \\
\hline IV & $3.99(2.69-5.90)$ & & $1.39(0.55-3.53)$ & \\
\hline Extrahepatic metastasis & & 0.031 & & 0.127 \\
\hline No & 1 & & 1 & \\
\hline Yes & $1.50(1.04-2.16)$ & & $1.95(0.83-4.60)$ & \\
\hline Hepatic metastasis & & $<0.001$ & & 0.015 \\
\hline No & 1 & & 1 & \\
\hline Yes & $2.79(1.91-4.06)$ & & $3.59(1.28-10.02)$ & \\
\hline Lymphatic or/and blood vessel invasion & & 0.009 & & 0.140 \\
\hline No & 1 & & 1 & \\
\hline Yes & $2.30(1.24-4.29)$ & & $2.04(0.79-5.27)$ & \\
\hline Nerve invasion & & 0.064 & & 0.884 \\
\hline No & 1 & & 1 & \\
\hline Yes & $1.76(0.97-3.21)$ & & $0.92(0.29-2.92)$ & \\
\hline Hepatoid Adenocarcinoma \# & & 0.102 & & 0.228 \\
\hline No & 1 & & & \\
\hline Yes & $1.68(0.90-3.12)$ & & & \\
\hline AFP $॥ \mathrm{HC}$ & & 0.375 & & 0.288 \\
\hline Low expression & 1 & & & \\
\hline High expression & $1.35(0.70-2.62)$ & & & \\
\hline
\end{tabular}

\#, 73 surgical patients out of 100 patients receiving second pathological diagnosis were included. 
TABLE 5 | Regression analysis for prognostic risk factors of APA-Gl liver metastasis.

\begin{tabular}{|c|c|c|c|c|}
\hline Variable & \multicolumn{2}{|c|}{ Single factor } & \multicolumn{2}{|c|}{ Multiple factors } \\
\hline$<60$ & 1 & & 1 & \\
\hline$\geq 60$ & $1.72(1.08-2.74)$ & & $2.13(0.68-6.65)$ & \\
\hline Sex & & 0.021 & & 0.123 \\
\hline Female & $0.55(0.33-0.91)$ & & $0.30(0.07-1.38)$ & \\
\hline Primary site & & 0.906 & & \\
\hline Stomach & 1 & & & \\
\hline Large intestine & $0.89(0.47-1.67)$ & & & \\
\hline Other & $0.86(0.27-2.72)$ & & & \\
\hline Pathological type & & 0.101 & & \\
\hline Adenocarcinoma & 1 & & & \\
\hline Signet ring cell carcinoma & $0.50(0.23-1.09)$ & & & \\
\hline Mucinous adenocarcinoma & $0.45(0.14-1.44)$ & & & \\
\hline Differentiation & & 0.741 & & \\
\hline Highly differentiated & 1 & & & \\
\hline Moderately differentiated & $1.34(0.18-9.94)$ & & & \\
\hline Poorly differentiated & $1.10(0.15-8.00)$ & & & \\
\hline pT & & 0.113 & & \\
\hline $1+2+3$ & 1 & & & \\
\hline$\geq 200$ & $2.24(1.48-3.39)$ & & $4.55(1.39-14.87)$ & \\
\hline Lymphatic or/and blood vessel invasion & & 0.013 & & 0.354 \\
\hline No & 1 & & 1 & \\
\hline Yes & $3.31(1.29-8.49)$ & & $1.69(0.56-5.14)$ & \\
\hline Nerve invasion & & 0.277 & & \\
\hline No & 1 & & & \\
\hline Yes & $1.6(0.69-3.73)$ & & & \\
\hline Hepatoid Adenocarcinoma" & & 0.029 & & 0.615 \\
\hline No & 1 & & 1 & \\
\hline Yes & $2.60(1.10-6.12)$ & & $0.75(0.35-2.27)$ & \\
\hline AFP IHC & & 0.626 & & \\
\hline Low expression & 1 & & & \\
\hline High expression & $1.22(0.54-2.77)$ & & & \\
\hline
\end{tabular}

\#, 73 surgical patients out of 100 patients receiving second pathological diagnosis were included.

adenocarcinomas and 25\% colorectal adenocarcinomas were initially diagnosed with liver metastasis, and approximately $50 \%$ of the patients with digestive adenocarcinoma eventually developed liver metastasis $(26,27)$. Our study found that in APA-GI, up to $58.2 \%$ of patients metastasized after the initial diagnosis, and the final metastasis rate reached $81.9 \%$. Among 


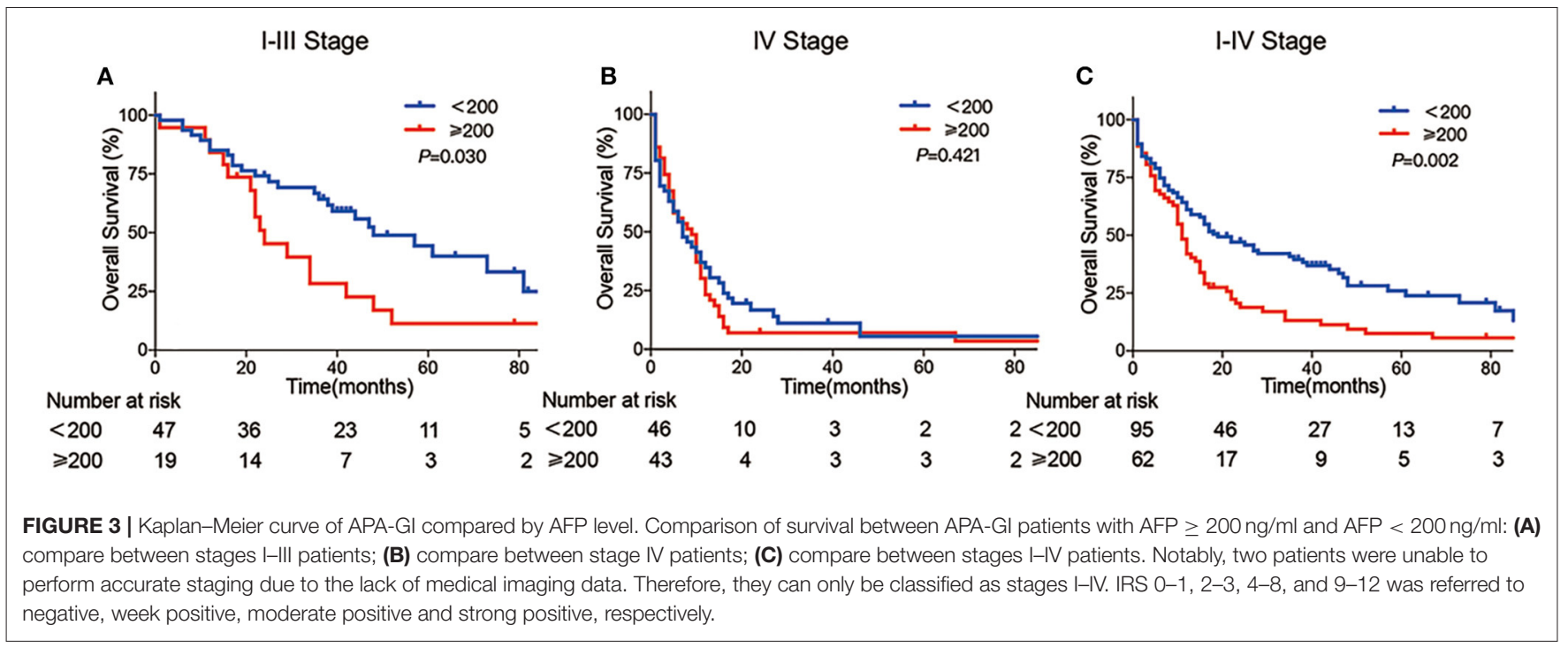

APA-GI, patients with liver metastasis at initial diagnosis were significantly more than those with CA-GI. Additionally, we performed a subgroup analysis by serum AFP with a bound of $200 \mathrm{ng} / \mathrm{ml}$. Consistent with previous studies, the AFP-H group was characterized by a higher rate of stage IV and liver metastasis, a larger tumor size, and a higher proportion of $\operatorname{HA}(5,28,29)$.

The prognosis of APA-GI was significantly worse than that of CA-GI. However, we did not observe a difference between stages I-III gastric and CRC patients, which may be related to $95.5 \%$ of stages I-III patients who underwent surgery. Early diagnosis of APA-GI and radical surgery may be an important treatment to improve prognosis. When APA-GI progressed to stage IV, the 5-year survival was only $6.3 \%$. We hypothesized that stage IV APA-GI patients were less sensitive to 5-Fu-based chemotherapy, similar to the $8.15 \%$ response rate of primary liver cancer to chemotherapy (30). Because of selection bias and recall bias in retrospective studies, the results of multivariate analyses varied among previous reports. Shoji and Feng et al. found that liver metastasis was the only independent prognostic factor for APA-GI $(6,31)$, whereas in other studies, TNM staging, serum AFP level, patient age, peritoneal seeding, lymph node metastasis, vascular invasion, Lauren classification, and AFP IHC results all became independent prognostic factors for APAGI $(29,32)$. Our study showed that patients with high AFP levels had a poor prognosis, whereas Cox regression analysis showed that liver metastasis was the only independent risk factor for APA-GI. We speculated that it might be due to the strong influence of liver metastasis on prognosis, thus masking the influence of high AFP level on prognosis. There were still several limitations in the present research. AFP has been reported not only in APA but also in yolk-sac tumors or some neuroendocrine tumors $(1,33,34)$. We asked two pathologists to recheck all $\mathrm{HE}$ sections and did not found any yolk sac tumor-like area or neuroendocrine component. However, for the other 77 patients without HE sections, we were unable to clarify whether AFP was produced only by APA. Whether APAGI is homogenous or heterogenous could not be concluded yet. Wang et al. had investigated the molecular features of HA of the stomach but showed limited homogenous (13). Besides, HA is theoretically not exactly the same as APA-GI. In future studies, we need more data from multiple centers to further study the clinicopathological characteristics of APA-GI, and basic research should be encouraged to investigate the pathogenesis of APA-GI.

\section{CONCLUSION}

The present study was the first research to systemically explored clinicopathological characteristics of APA-GI. APA-GI occurs through the whole GI tract with a significantly worse prognosis than CA-GI. APA-GI should be regarded as one kind of disease for its similar clinicopathological characteristics within patients.

\section{DATA AVAILABILITY STATEMENT}

The raw data supporting the conclusions of this article will be made available by the authors, without undue reservation.

\section{AUTHOR CONTRIBUTIONS}

$\mathrm{X}-\mathrm{XK}, \mathrm{X}-\mathrm{LL}$, and X-MX wrote the first draft of the manuscript. All authors commented on previous versions of the manuscript. All authors contributed to the study's conception and design. All authors performed material preparation, data collection, and analysis. All authors commented on previous versions of the manuscript. All authors read and approved the final manuscript. 


\section{FUNDING}

This work was supported by the Key Technology Research and Development Program of Zhejiang Province (2017C03017), the National Key R\&D Program of China (2017YFC0908200), and the National Natural Science Foundation of China (81672916, 11932017, and 82072360).

\section{ACKNOWLEDGMENTS}

We gratefully thank Hai-Ting Xie, Zhan-Huai Wang, Shao-Jun $\mathrm{Yu}$, Ye-Ting $\mathrm{Hu}$, Dong $\mathrm{Xu}$, and Li-Feng Sun for helping perform the operations.

\section{REFERENCES}

1. El-Bahrawy M. Alpha-fetoprotein-producing non-germ cell tumours of the female genital tract. Eur $J$ Cancer. (2010) 46:1317-22. doi: 10.1016/j.ejca.2010.01.028

2. Yamamura N, Fugo K, Kishimoto T. Forkhead box protein A2, a pioneer factor for hepatogenesis, is involved in the expression of hepatic phenotype of alpha-fetoprotein-producing adenocarcinoma. Pathol Res Pract. (2017) 213:1082-8. doi: 10.1016/j.prp.2017.07.024

3. Ren $\mathrm{F}$, Weng $\mathrm{W}$, Zhang $\mathrm{Q}$, Tan $\mathrm{C}$, Xu $\mathrm{M}$, Zhang $\mathrm{M}$, et al. Clinicopathological features and prognosis of AFP-producing colorectal cancer: a single-center analysis of 20 cases. Cancer Manag Res. (2019) 11:4557-67. doi: 10.2147/CMAR.S196919

4. Wang D, Li C, Xu Y, Xing Y, Qu L, Guo Y, et al. Clinicopathological characteristics and prognosis of alpha-fetoprotein positive gastric cancer in Chinese patients. Int J Clin Exp Pathol. (2015) 8:6345-55.

5. Wang YK, Zhang XT. [AFP-producing gastric cancer and hepatoid gastric cancer]. Zhonghua Zhong Liu Za Zhi. (2017) 39:801-7. doi: 10.3760/cma.j.issn.0253-3766.2017.11.001

6. Feng Y, Li Y, Dai W, Mo S, Li Q, Cai S. Clinicopathologic features and prognostic factors in alpha-fetoprotein-producing colorectal cancer: analysis of 78 cases. Cell Physiol Biochem. (2018) 51:2052-64. doi: 10.1159/000495824

7. Inagawa S, Shimazaki J, Hori M, Yoshimi F, Adachi S, Kawamoto T, et al. Hepatoid adenocarcinoma of the stomach. Gastric Cancer. (2001) 4:4352. doi: 10.1007/s101200100016

8. Supriatna Y, Kishimoto T, Uno T, Nagai Y, Ishikura H. Evidence for hepatocellular differentiation in alpha-fetoprotein-negative gastric adenocarcinoma with hepatoid morphology: a study with in situ hybridisation for albumin mRNA. Pathology. (2005) 37:211-5. doi: 10.1080/00313020500099221

9. Tamai Y, Kobayashi K, Haruki Y, Yada T, Urade S, Harada T, et al. [A case of alpha-fetoprotein-producing fetal gut-like small intestinal cancer]. Nihon Shokakibyo Gakkai Zasshi. (2013) 110:833-8. doi: 10.11405/nisshoshi.110.833

10. Gushima R, Narita R, Shono $T$, Naoe H, Yao T, Sasaki Y. Esophageal adenocarcinoma with enteroblastic differentiation arising in ectopic gastric mucosa in the cervical esophagus: a case report and literature review. J Gastrointestin Liver Dis. (2017) 26:193-7. doi: 10.15403/jgld.2014.1121.262.gus

11. Takahashi N, Aoyama F, Hiyoshi M, Kataoka H, Sawaguchi A. Establishment and biological characterization of a novel cell line derived from hepatoid adenocarcinoma originated at the ampulla of vater. Int J Oncol. (2014) 44:1139-45. doi: 10.3892/ijo.2014.2282

12. Ooi A, Nakanishi I, Sakamoto N, Tsukada Y, Takahashi Y, Minamoto $\mathrm{T}$, et al. Alpha-fetoprotein (AFP)-producing gastric carcinoma. Is it hepatoid differentiation? Cancer. (1990) 65:17417. doi: 10.1002/1097-0142(19900415)65:8<1741::AID-CNCR28206508 $14>3.0 . \mathrm{CO} ; 2-3$

13. Wang Y, Sun L, Li Z, Gao J, Ge S, Zhang C, et al. Hepatoid adenocarcinoma of the stomach: a unique subgroup with distinct

\section{SUPPLEMENTARY MATERIAL}

The Supplementary Material for this article can be found online at: https://www.frontiersin.org/articles/10.3389/fonc. 2021.635537/full\#supplementary-material

Supplementary Figure 1 | Kaplan-Meier curve of HA positive and HA negative within APA-GI Comparison of survival between HA positive and HA negative within 73 APA-Gl patients who received operation.

Supplementary Figure 2 | Typical immunohistochemical staining images of APA-Gl a, Negative; b, Weakly positive; c, Moderately positive; $d$, Strongly positive.

Supplementary Figure 3 | Kaplan-Meier curve of AFP-/HA- between the other patients within APA-Gl. Comparison of survival between AFP-/HA- and the other patients within 73 APA-Gl patients who received operation.

clinicopathological and molecular features. Gastric Cancer. (2019) 122:183-192. doi: 10.1007/s10120-019-00965-5

14. Liu M, Liu B, Zhou Y, Quan X, Liu B, Dong L. AFP-producing lung squamous carcinoma. QJM. (2016) 109:813-4. doi: 10.1093/qjmed/hcw179

15. Acosta AM, Pins MR. Hepatoid carcinoma of the ovary: clinical, histopathologic, and immunophenotypic features. Arch Pathol Lab Med. (2019) 143:883-9. doi: 10.5858/arpa.2017-0485-RS

16. Blandamura S, Vendraminelli R, Aversa S, Fedrigo M. Collecting duct carcinoma of kidney producing alpha-fetoprotein. Eur J Surg Oncol. (2005) 31:1039-41. doi: 10.1016/j.ejso.2005.07.001

17. Sun W, Liu B, Chen J, Gong P, Wu X, Liu C, et al. Novel characteristics of alpha-fetoprotein (AFP)-producing gastric cancer. Oncotarget. (2017) 8. 101944-51. doi: 10.18632/oncotarget.22109

18. Takashima H, Kimura H, Nakamura H, Myojo S, Okuyama Y, Sugeta N, et al. [A case of AFP producing endocrine cell carcinoma of the duodenum]. Nihon Shokakibyo Gakkai Zasshi. (2002) 99:798-802. doi: 10.11405/nisshoshi1964. 99.798

19. Nagtegaal ID, Odze RD, Klimstra D, Paradis V, Rugge M, Schirmacher P, et al. The 2019 WHO classification of tumours of the digestive system. Histopathology. (2020) 76:182-188. doi: 10.1111/his.13975

20. Wang YK, Shen L, Jiao X, Zhang XT. Predictive and prognostic value of serum AFP level and its dynamic changes in advanced gastric cancer patients with elevated serum AFP. World J Gastroenterol. (2018) 24:26673. doi: $10.3748 /$ wjg.v24.i2.266

21. Shimakata T, Kamoshida S, Kawamura J, Ogane N, Kameda Y, Yanagita E, et al. Immunohistochemical expression profiles of solute carrier transporters in alpha-fetoprotein-producing gastric cancer. Histopathology. (2016) 69:81221. doi: 10.1111/his.13004

22. Li Z, Hou X, Chen J, Sun H, Mi Y, Sui Y, et al. Efficacy and safety of SOX chemotherapy with or without surgery in AFP-producing advanced gastric cancer. Oncol Lett. (2017) 14:579-86. doi: 10.3892/ol.2017. 6240

23. He X, Wu W, Lin Z, Ding Y, Si J, Sun LM. Validation of the american joint committee on cancer (AJCC) 8th edition stage system for gastric cancer patients: a population-based analysis. Gastric Cancer. (2018) 21:391400. doi: 10.1007/s10120-017-0770-1

24. Van Cutsem E, Nordlinger B, Cervantes A, Group EGW. Advanced colorectal cancer: ESMO clinical practice guidelines for treatment. Ann Oncol. (2010) 21(Suppl. 5):v93-7. doi: 10.1093/annonc/mdq222

25. Bernards N, Creemers GJ, Nieuwenhuijzen GA, Bosscha K, Pruijt JF, Lemmens VE. No improvement in median survival for patients with metastatic gastric cancer despite increased use of chemotherapy. Ann Oncol. (2013) 24:3056-60. doi: 10.1093/annonc/mdt401

26. Song JC, Ding XL, Zhang Y, Zhang X, Sun XH. Prospective and prognostic factors for hepatic metastasis of gastric carcinoma: a retrospective analysis. $J$ Cancer Res Ther. (2019) 15:298-304. doi: 10.4103/jcrt.JCRT

27. Nordlinger B, Sorbye H, Glimelius B, Poston GJ, Schlag PM, Rougier $\mathrm{P}$, et al. Perioperative chemotherapy with FOLFOX4 and surgery versus surgery alone for resectable liver metastases from colorectal cancer (EORTC 
intergroup trial 40983): a randomised controlled trial. Lancet. (2008) 371:1007-16. doi: 10.1016/S0140-6736(08)60455-9

28. Liu X, Cheng Y, Sheng W, Lu H, Xu Y, Long Z, et al. Clinicopathologic features and prognostic factors in alpha-fetoprotein-producing gastric cancers: analysis of 104 cases. J Surg Oncol. (2010) 102:249-55. doi: 10.1002/jso.21624

29. Lin HJ, Hsieh YH, Fang WL, Huang KH, Li AF. Clinical manifestations in patients with alpha-fetoprotein-producing gastric cancer. Curr Oncol. (2014) 21:e394-9. doi: 10.3747/co.21.1768

30. Qin S, Bai Y, Lim HY, Thongprasert S, Chao Y, Fan J, et al. Randomized, multicenter, open-label study of oxaliplatin plus fluorouracil/leucovorin versus doxorubicin as palliative chemotherapy in patients with advanced hepatocellular carcinoma from Asia. J Clin Oncol. (2013) 31:35018. doi: 10.1200/JCO.2012.44.5643

31. Hirajima S, Komatsu S, Ichikawa D, Kubota T, Okamoto K, Shiozaki A, et al. Otsuji, Liver metastasis is the only independent prognostic factor in AFP-producing gastric cancer. World J Gastroenterol. (2013) 19:605561. doi: 10.3748/wjg.v19.i36.6055

32. Yang J, Wang R, Zhang W, Zhuang W, Wang M, Tang C. Clinicopathological and prognostic characteristics of hepatoid adenocarcinoma of the stomach.
Gastroenterol Res Pract 2014. (2014) 2014:140587. doi: 10.1155/2014/1 40587

33. Wang CY, Lin JC, Li YF, Yang CW. Alpha-fetoprotein producing pancreatic neuroendocrine tumour. QJM. (2020) 113:565-6. doi: 10.1093/qjmed/hcaa018

34. Zhu X, Yong H, Zhang L, Huang Y, Zheng J, Liu C, et al. Pure alphafetoprotein-producing neuroendocrine carcinoma of the pancreas: a case report. BMC Gastroenterol. (2015) 15:16. doi: 10.1186/s12876-015-0246-x

Conflict of Interest: The authors declare that the research was conducted in the absence of any commercial or financial relationships that could be construed as a potential conflict of interest.

Copyright (C) 2021 Kong, Li, Tian, Ye, Xu, Liu, Yang, Zhang, Mei, Wen, Xiao, Li, Ding and $\mathrm{Li}$. This is an open-access article distributed under the terms of the Creative Commons Attribution License (CC BY). The use, distribution or reproduction in other forums is permitted, provided the original author(s) and the copyright owner(s) are credited and that the original publication in this journal is cited, in accordance with accepted academic practice. No use, distribution or reproduction is permitted which does not comply with these terms. 